Vol. 21, 2019/e39

\title{
La sintonización en la formación inicial docente. Una mirada desde Chile
}

\author{
Tuning in the Initial Training of Teachers. A View from Chile
}

\author{
Donatila Ferrada $\left(^{*}\right)$ donatila.ferrada@gmail.com \\ (*) Universidad Católica del Maule \\ (Recibido: 31 de julio de 2017; Aceptado para su publicación: 12 de diciembre de 2017)
}

Cómo citar: Ferrada, D. (2019). La sintonización en la formación inicial docente. Una mirada desde Chile. Revista Electrónica de Investigación Educativa, 21, e39, 1-14. doi:10.24320/redie.2019.21.e39.2113

\section{Resumen}

La formación inicial docente configura un ámbito de especial interés para la investigación en Chile, ya que se ha impulsado una política conocida como programa de Mejoramiento de la Calidad de la Educación Superior (Mecesup), dirigida a renovar la educación terciaria en dos sentidos: uno vincula la asignación de recursos con resultados de desempeño, y otro con resultados de aprendizaje y demostración de competencias sintonizadas con el mundo. De este contexto, el objetivo de la investigación es determinar la sintonía producto de la renovación curricular entre carreras de pedagogía en educación general básica con y sin programa Mecesup, en cuanto al diseño organizado en competencias generales y específicas. En términos metodológicos, se construyeron tres estudios de casos situados en la zona centro-sur del país, y mediante un estudio documental de los planes de estudios de las carreras se establecieron relaciones entre todos los casos. Se concluye que no existe sintonía entre las carreras estudiadas independientemente de contar o no con Mecesup.

Palabras clave: Currículum, política educativa, formación inicial, competencias, sintonización.

\begin{abstract}
Initial teacher training sets an area of particular interest for research in Chile, as it has promoted a policy known as Mecesup aimed at renewing tertiary education in two ways, one that links resource allocation with performance results, and another, linking training with learning outcomes and demonstration of skills attuned to the world. In this context, the main objective is to determine the harmony product of the curricular renovation between elementary teaching study plans with and without Mecesup program, as organized general and specific skills in design. In terms of methodology, three case studies located in the south central part of the country are built, and through an informed research of the study plans of the teaching programmes relations among the three cases were established. We conclude that there is no harmony between the teaching programmes studied regardless of having or not having Mecesup project.
\end{abstract}

Keywords: Curriculum, education policy, initial training, skills, tuning. 


\section{Introducción}

Chile cuenta con el sistema educativo más segregado entre las 34 naciones miembros de la OCDE, vinculando alta desigualdad socioeconómica con resultados académicos (Valenzuela, Bellei y De los Ríos, 2011). Este eje problemático nacional, entre otras variables, se identifica como crucial en la formación inicial del profesorado en el país, cuestión que ha obligado a incluirla como tema de la agenda política nacional durante las últimas décadas. Esto ha implicado la promoción de cambios en la formación docente por medio de dotación de recursos económicos a las instituciones formadoras, a través de diversos programas con fondos concursables, entre ellos, el Proyecto Fortalecimiento de la Formación Inicial Docente (PFFID) iniciado en 1997, el cual fue desestimado tempranamente. Casi en forma paralela a éste se implementó el Programa de Mejoramiento de la Calidad de la Educación Superior (Mecesup), que el Gobierno de Chile convino con el Banco Mundial para el mejoramiento de la calidad de la educación terciaria, cuyo primer concurso del Fondo Competitivo fue en 1999, año en que se transitó de un sistema de asignación de recursos basados en criterios históricos, sin rendición de cuentas públicas, a un nuevo escenario de asignación por resultados (Mineduc, 2015). Si bien este programa se encuentra vigente, ha sido redefinido en 2012, como sigue:

El Programa Mecesup2 permitió sentar las bases para: i) El desarrollo y escalamiento de un sistema de financiamiento incremental basado en convenios de desempeño (CD); ii) La implementación inicial en el ámbito nacional de una nueva arquitectura curricular para la educación superior basada en resultados de aprendizaje y demostración de competencias y sintonizada con el mundo; iii) La definición e implementación inicial de un sistema para la transferencia de créditos académicos (scT-Chile, compatible con el ECTS europeo) que permita la movilidad estudiantil nacional e internacional. (Mineduc, 2013, p. 13).

Así se cambia la orientación de la innovación en la educación terciaria, desde una pensada por los sujetos desde sus instituciones, por una regulada desde el Estado hacia las instituciones. De acuerdo con Hord (1987), este tipo de innovación correspondería a una propuesta organizativa desde arriba a fin de dar cuenta de demandas identificadas en el sistema de forma más rápida y eficaz. En correspondencia con ello, puede caracterizarse como una innovación de tipo administrativo toda vez que resulta normativa (Marcelo, 2000) en cuanto a que el énfasis está en la regulación entre los fondos y los productos a alcanzar, y porque la fase de iniciación innovadora es promovida por el Estado y no por las instituciones formadoras (Estebaranz, 1994).

En este nuevo escenario nacional, si bien la innovación es más fácil de implementar cuando viene desde la autoridad, se advierte que:

Aun en este caso, el esfuerzo del cambio sólo es eficaz mientras alguien lo está empujando. El éxito depende del esfuerzo continuo, del entusiasmo de las ideas, y de las iniciativas de las personas que las han de llevar a la práctica (Mingorance y Estebaranz, 2009, p. 180)

En correspondencia con lo anterior, la "nueva arquitectura curricular" asumida por la política Mecesup en Chile ha encontrado gran eco en las instituciones de educación superior, es decir, su implementación efectivamente ha sido más rápida, y los equipos impulsores mientras cuentan con los apoyos económicos al adjudicarse estos proyectos renuevan los currículos de formación inicial, previa condición del Estado, eso sí, de adscripción al Modelo Tuning Europeo (Mineduc, 2013), en versión de lo que se conoce como proyecto Tuning-Latinoamérica (Bravo, 2007).

De esta forma, el modelo basado en competencias se configuró en la única opción para postular a este tipo de fondos estatales, al mismo tiempo que se constituyó en un modelo legítimo y actualizado desde el cual reorganizar "las estructuras y el contenido de los estudios" (González y Wagenaar, 2003). Así, se concretó una profunda renovación curricular de todas las carreras universitarias a nivel de país, incluidas las de pedagogía, cambios que tuvieron como característica principal las asesorías internacionales de expertos diseñadores del currículum basado en competencias, encargados de orientar la sintonización a nivel de estructuras curriculares y de sistema de créditos transferibles que permitieran la movilidad 
estudiantil a nivel nacional e internacional, tal como definía la política del Mecesup.

De estos grandes cambios producidos a nivel de formación universitaria en Chile, se espera, por una parte, una mayor movilidad estudiantil nacional e internacional, y por otra, una sintonización del currículum de formación inicial. Respecto del primer cambio, los datos sistematizados a la fecha reportan una escasísima movilidad (menos del $1 \%$ en un período de tres años) de estudiantes están accediendo a este beneficio tanto a nivel nacional como internacional (Del Valle, Kri y Segovia, 2013). Y respecto del segundo, aún no existen estudios sistematizados que develen la sintonización que se persigue, ni tampoco está claro qué significa o qué se entiende por ello, por lo mismo, resulta necesario profundizar en este ámbito.

Una revisión a la literatura permite comprender la idea de sintonía en la formación de profesores propuesta por la Comunidad Europea. Así, en la formación docente -ámbito específico que motiva este trabajo-, la sintonización implicaría, en primer término, "una definición compartida de habilidades y conocimiento docente, como marco para guiar la formación y el desarrollo profesional a lo largo de la carrera". (Caena, 2011, p. 2).

En segundo término y siguiendo a Caena (2011), la sintonía en este marco de referencia común requiere de la articulación de tres amplias áreas de competencias para formar el futuro docente o competencias generales, como también se les denomina. La primera, trabajar con otros (integra valores de inclusión, desarrollo del potencial de cada alumno, habilidades interpersonales y cooperativas y conocimiento psicológico-pedagógico). La segunda, trabajar con conocimiento, tecnología e información (incluye capacidad de recuperar, gestionar y analizar críticamente varios tipos de información, habilidades digitales aplicadas con fines profesionales, junto con habilidades pedagógicas y de enseñanza). Y la tercera, trabajar en y con la sociedad (que los profesores actúen como profesionales responsables en las comunidades educativas locales y con diferentes actores, y promuevan el desarrollo de los estudiantes como ciudadanos con responsabilidades globales, alentando disposiciones y actitudes hacia la cooperación y la movilidad, el diálogo intercultural y el respeto).

En tercer término incluye un conjunto de competencias especializadas de las materias siguientes: enseñar en clases heterogéneas, utilizar las TIC, enseñar competencias transversales, crear escuelas seguras y atractivas, desarrollar culturas y actitudes propias de la práctica reflexiva, investigación, innovación, colaboración y aprendizaje autónomo. Todas ellas identificadas como las "ocho claves requeridas en un mundo cambiante, en particular, la metacompetencia de aprender a aprender (ajustándose al cambio, gestionando y seleccionando grandes flujos de información)" (Caena, 2011, pp. 2-4).

Una primera dificultad observada a nivel práctico en Chile, respecto de la sintonización de la "arquitectura curricular" desde el modelo basado en competencias liderado por los asesores internacionales, es que éstos no fueron portadores de modelos unificadores. Este hecho, ya evidenciado en otras partes del mundo a través de investigaciones que demostraban que mientras unos asesores adscribían a corrientes teóricas más funcionalistas otros optaban por otras más sociocontructivistas. Los primeros a través de una construcción curricular de secuenciación detallada y compartimentalizada de cada competencia, los segundos, a la base de la pretensión de superar la visión anterior, optaron por establecer una relación con la adaptación de la persona y su interacción con la situación y el contexto en que se desempeñará el futuro profesional, construyendo así competencias más situadas y contextualizadas (Jonnaert, Barrette, Masciotra y Yaya, 2008).

Estos antecedentes inducen a pensar que es probable que esta dicotomía curricular de los asesores internacionales de la renovación curricular en la formación inicial docente también fuera trasladada a Chile. Esto permite problematizar tanto la sintonización que persigue Mecesup, como a cuál de estas visiones han adscrito los procesos de renovación curricular de las instituciones formadoras de profesores. En términos concretos, realizando una panorámica general del currículum renovado bajo el programa Mecesup, la mayoría de ellos observan una estructura común en cuanto que organizan los planes y programas en torno a la construcción de competencias generales y específicas. Pero, ¿qué ocurre a nivel de contenidos de esas competencias generales y específicas? En Chile no existen estudios sistematizados 
que reporten este nivel de análisis en el país, es decir, la sintonización sigue siendo un supuesto que podría estar quedando sólo en la forma. Por otra parte, la renovación curricular que se está llevando a cabo, ¿superará la visión funcionalista del currículum y alcanzará una visión constructivista de la misma?, como lo sugiere la literatura especializada (Aneas, 2009; De La Vega, 2015; Jonnaert, Barrette, Masciotra y Yaya, 2008; Márquez, 2012) sobre todo en relación con la inclusión de temáticas sobre diversidad de los contextos social, cultural y emocional que forman parte del escenario de desempeño cotidiano del trabajo pedagógico de enseñanza y aprendizaje (Caena, 2011; Gallego y Rodríguez, 2007; Levine, 2006; Noguera, 2003; Townsend, 2007; Zeichner, 2008).

Hoy, a más de una década de que egresaran los primeros docentes bajo esta renovación curricular supuestamente sintonizada, se hace cada vez más urgentes realizar estudios que permitan observar estas cuestiones. Desde este escenario, y si bien resultaría interesante realizar un estudio a nivel nacional, esta investigación se circunscribió a la zona centro-sur del país, concentrándose en la pregunta: ¿Existe sintonía entre las competencias generales y específicas de los currículos renovados de pedagogía en educación general básica en instituciones universitarias con y sin proyecto Mecesup?

\section{Método}

De acuerdo con la clasificación de Guba y Lincoln (2012), esta investigación se adscribe al paradigma participativo que comprende la realidad como interacción entre lo subjetivo-objetivo, como una cocreación entre un universo dado y la construcción social de los sujetos; es decir, de naturaleza participativa, donde los saberes experienciales de los sujetos, proposiciones y prácticas interactúan colaborativamente con aquellos presentes en el universo, que configuran comunidades de investigación que igualan la jerarquía epistémica entre quienes asumen el rol de investigadores de quienes lo hacen en calidad de investigados. En correspondencia con este paradigma, se optó por asumir el enfoque de investigación dialógico-kishu kimkelay ta che (Astorga 2017; Ferrada y Del Pino, 2017; Ferrada et al, 2014; George-Nascimento, 2015) dado que éste se focaliza en construir conocimiento científico de carácter colectivo, situado y territorializado, a partir de la conformación de una comunidad de investigación, la cual decide qué, por qué y cómo investigar. En el caso de esta investigación, se constituyó una comunidad de investigación conformada por 8 personas (2 académicos formadores de profesores, 2 profesores con desempeño laboral en escuelas de la zona y 4 estudiantes de Pedagogía). Es esta comunidad de investigación la que desarrolló todos los procesos investigativos de forma colectiva y desde diferentes configuraciones de sentido, con lo cual se da mayor validez a lo que se construye como conocimiento.

Esta comunidad de investigación, una vez que acordó la pregunta, definió los siguientes objetivos de investigación: 1) Descubrir unidades de análisis en competencias generales y específicas de carreras de pedagogía básica con y sin Mecesup que contengan significados comunes/equivalentes entre ellas a fin de determinar la existencia de sintonía entre estas carreras; 2) Identificar la estructura de las competencias generales y específicas de cada carrera estudiada a fin de determinar la existencia de sintonía entre estas carreras; 3 ) Identificar la corriente curricular a partir de las unidades de análisis y el tipo de estructura de cada competencia. Enseguida, la comunidad de investigación decidió que estos objetivos fueran respondidos desde la construcción de estudios de casos múltiples (Stake, 1998), cuya finalidad es comprender algo más que el caso particular -en esta ocasión la sintonización producida por la política Mecesup en la formación docente.

Para tales efectos se construyeron 3 casos $^{1}$, en adelante identificados como Caso A: Currículum renovado de pedagogía en educación general básica de una institución universitaria con Mecesup; Caso B: Currículum renovado de Pedagogía en educación general básica, institución universitaria con Mecesup; Caso C: Currículum renovado de Pedagogía en educación general básica, institución universitaria sin Mecesup. Las fuentes usadas fueron de carácter documental, y consistieron en los planes y programas de

\footnotetext{
${ }^{1}$ Usando como criterios de selección los siguientes: Carrera de pedagogía básica; con currículum renovado con Proyecto Mecesup; con currículum renovado sin proyecto Mecesup; carreras que pertenezcan a Instituciones formadoras de la zona centro-sur de Chile; equipos de gestión curricular dispuestos a facilitar la información para el estudio.
} 
estudio con currículum renovado de la carrera de Pedagogía en educación general básica ya identificadas en cada caso.

Para dar cuenta de todos los objetivos se usó el procedimiento de construcción de conocimiento colectivo discurso dialógico propio del enfoque de investigación dialógica-kishu kemkelay ta che, entendido como una interpretación intersubjetiva entre los miembros de una comunidad de investigación de discursos orales y/o unidades de texto (párrafos), en este caso, con base en las unidades de análisis de contenido de texto, dado el carácter documental de la información con la que se trabajó.

En consideración a este carácter intersubjetivo del proceso investigativo, la comunidad de investigación decidió para el primer objetivo, la construcción de unidades de análisis. Para el segundo objetivo acordó adoptar como definición de estructura de competencia a aquella capaz de cumplir con las tres grandes dimensiones que configuran una competencia cualquiera: saber (conocimientos), saber hacer (habilidades) y ser (actitudes) (Rué y Martínez, 2005; Caena, 2011). Finalmente, para el tercer objetivo, se realizó un análisis de segundo nivel a partir de los indicadores de estructura de la competencia, unidad de análisis y característica de la competencia, todos ellos reportados por los dos objetivos anteriores.

\section{Resultados}

\subsection{La sintonía en competencias generales en los estudios de caso}

La pregunta sobre la sintonía entre las competencias generales de los currículos renovados de Pedagogía en educación general básica en instituciones universitarias con y sin proyecto Mecesup sólo pudo ser respondida desde los casos A y B con Mecesup, pues ellos dan cuenta de un diseño curricular en correspondencia con el modelo Tuning basado en competencia, definiendo competencias generales como eje principal de la organización del currículum. En cambio el caso C (sin Mecesup) no se organiza en torno a este diseño, por cuanto no hay información para evaluar la sintonía a este nivel.

Los resultados de los casos A y B se presentan en la tabla I, y recogen tanto las unidades de análisis (Objetivo 1) como la estructura identificada en cada competencia (Objetivo 2). 
Tabla I. Competencias generales en los casos A y B

\begin{tabular}{|c|c|c|c|c|}
\hline \multirow[t]{2}{*}{$\mathrm{N}^{\circ}$} & \multicolumn{2}{|c|}{ Caso A (Mecesup) } & \multicolumn{2}{|c|}{ Caso B (Mecesup) } \\
\hline & Unidad de Análisis & $\begin{array}{l}\text { Estructura de la } \\
\text { competencia }\end{array}$ & Unidad de Análisis & $\begin{array}{l}\text { Estructura de la } \\
\text { competencia }\end{array}$ \\
\hline 1 & $\begin{array}{l}\text { Actitud de autoaprendizaje y } \\
\text { actualización teórica y } \\
\text { tecnológica para el ejercicio } \\
\text { de la profesión. }\end{array}$ & $\begin{array}{l}\text { SER; SABER; } \\
\text { SABER HACER }\end{array}$ & Autoaprendizaje & SER \\
\hline 2 & $s / i$ & $\mathrm{~s} / \mathrm{i}$ & Actualización & SABER HACER \\
\hline 3 & $\mathrm{~s} / \mathrm{i}$ & $s / i$ & Usa tic & SABER HACER \\
\hline 4 & $s / i$ & $\mathrm{~s} / \mathrm{i}$ & Adaptación al entorno & $\begin{array}{l}\text { SER; SABER } \\
\text { HACER }\end{array}$ \\
\hline 5 & $\begin{array}{l}\text { Ciudadanía para compromiso } \\
\text { con medio social, natural y } \\
\text { cultural }\end{array}$ & $\begin{array}{l}\text { SER; SABER; } \\
\text { HACER-SABER }\end{array}$ & $\begin{array}{l}\text { Ética y moral basada en } \\
\text { antropología cristiana }\end{array}$ & SER; SABER \\
\hline 6 & $\mathrm{~s} / \mathrm{i}$ & $s / i$ & Diálogo fe-razón & SER; SABER \\
\hline 7 & $\begin{array}{l}\text { Intercambio con otras } \\
\text { disciplinas y resguardo ético }\end{array}$ & $\begin{array}{l}\text { SABER HACER; } \\
\text { SABER; SER }\end{array}$ & Integra equipo interdisciplinar & $\begin{array}{l}\text { SER; SABER } \\
\text { HACER }\end{array}$ \\
\hline 8 & $\begin{array}{l}\text { Innova, decide en contexto } \\
\text { laboral }\end{array}$ & $\begin{array}{l}\text { SABER HACER; } \\
\text { SER }\end{array}$ & Innova & SABER HACER \\
\hline 9 & $\mathrm{~s} / \mathrm{i}$ & $s / i$ & Decide & SABER HACER \\
\hline 10 & $\begin{array}{l}\text { Comunicación oral y escrita } \\
\text { en dos idiomas para } \\
\text { interactuar socialmente }\end{array}$ & $\begin{array}{l}\text { SABER HACER; } \\
\text { SABER; SER }\end{array}$ & $\begin{array}{l}\text { Comunicación oral y escrita } \\
\text { efectiva en castellano }\end{array}$ & $\begin{array}{l}\text { SABER HACER; } \\
\text { SABER }\end{array}$ \\
\hline 11 & $\mathrm{~s} / \mathrm{i}$ & $s / i$ & $\begin{array}{l}\text { Comunicación oral y escrita en } \\
\text { inglés }\end{array}$ & $\begin{array}{l}\text { SABER HACER; } \\
\text { SABER }\end{array}$ \\
\hline
\end{tabular}

Notas. Codificación: s/i: sin información; TIc: Tecnologías de la Información y Comunicación.

A nivel general los resultados evidencian una diferencia numérica entre un caso y otro; el caso A define 5 competencias generales $(1,5,7,8$ y 10$)$, mientras que el caso B define 11 . Sin embargo, las competencias definidas por el caso A encuentran correspondencia en una o más competencias de caso B, es decir, mientras el caso A define competencias más integradoras, el caso B define competencias muy fragmentadas.

En cuanto a la relación con las unidades de análisis, los resultados permiten observar que la competencia 1 del caso $A$ encuentra relación con las competencias 1, 2, 3 y 4 del caso B. Lo mismo ocurre con la competencia 8 del caso A, con 8 y 9 del caso B; y la competencia 10 del caso A, con 10 y 11 del caso B. Sin embargo, esta equivalencia de significado no es plena, pues mientras en el caso A sitúa el significado en el contexto (laboral, social), en el caso B lo hace en el sujeto. En cambio, las competencias 7 en ambos casos encuentran relación sólo entre ellas. A diferencia de la competencia 5 del caso A, con 5 y 6 del caso B, que no observan equivalencia alguna en cuanto a significados en unidades de análisis que se comparan, sin embargo, equivalen en que ellas definen el sello institucional.

Respecto de la estructura de cada competencia, se evidencia que mientras el caso A de las 5 competencias que declara, 4 de ellas cumplen con las tres dimensiones (saber, el saber hacer y el ser). En cambio, B define 11 competencias, de las cuales, ninguna de ellas cumple el requisito de las tres dimensiones, es más, la mitad de ellas cumple dos y la otra mitad sólo una, constituyéndose el "saber hacer" (habilidades) como la dimensión central en la construcción de cada competencia (en 8 de 11).

El objetivo 3 que persigue identificar las corrientes curriculares en los diseños renovados de las carreras de Pedagogía general básica, desde los datos reportados en los objetivos 1 y 2 (ver tabla II). 
Tabla II. Corrientes curriculares en los casos A y B

\begin{tabular}{l|l|l}
\hline \multicolumn{1}{c|}{ Indicadores } & \multicolumn{1}{|c}{ Caso A con Mecesup } & \multicolumn{1}{c}{ Caso B con Mecesup } \\
\hline $\begin{array}{l}\text { Estructura de la competencia } \\
\text { (dimensiones) }\end{array}$ & $\begin{array}{l}\text { Cumple } \\
\text { Presencia de tres dimensiones } \\
\text { (ser-saber hacer-saber) }\end{array}$ & $\begin{array}{l}\text { No cumple } \\
\text { Presencia de una y dos dimensiones } \\
\text { (el saber hacer es el eje central) }\end{array}$ \\
\hline Unidad de análisis & $\begin{array}{l}\text { Unidades de significados generales } \\
\text { ajustadas a distintos contextos } \\
\text { vinculados con la profesión. }\end{array}$ & $\begin{array}{l}\text { Unidades de significados parciales } \\
\text { sin contexto alguno }\end{array}$ \\
\hline $\begin{array}{l}\text { Características de las } \\
\text { competencias }\end{array}$ & $\begin{array}{l}\text { Integrales } \\
\text { Genéricas } \\
\text { Situadas en algún contexto }\end{array}$ & $\begin{array}{l}\text { Fragmentadas } \\
\text { Acotadas } \\
\text { Centradas en algún objeto }\end{array}$ \\
\hline
\end{tabular}

Estos hallazgos permiten vincular al caso A con la corriente curricular de orientación constructivista, toda vez que las características de las competencias son integrales, genéricas y situadas en contexto, de la misma forma los significados que se construyen resultan coincidentes con ellas, y las competencias dan cuenta de una construcción que integra el saber, con el saber hacer y el ser. Por el contrario, el caso B, cuyas competencias se caracterizan por ser fragmentadas, acotadas y centradas en algún objeto, resultan coincidentes con la corriente curricular de orientación funcionalista (Joannert et al., 2008), refrendado por las unidades de significados parciales y descontextualizadas y sin dar cuenta de la estructura mínima exigida de una competencia (Rué y Martínez, 2005).

Así también, desde un análisis del modelo basado en competencia, sólo el caso A cumple parcialmente con uno de los tres ejes del marco referencial común exigido por Tuning, a saber, trabajar con conocimiento, tecnología e información (Caena, 2011) pues logra vincular este ámbito con habilidades pedagógicas, aunque con ello no alcance a dar cuenta de la sintonía. Por consiguiente, no resulta posible demostrar sintonía pues no existe una definición compartida de habilidades y conocimiento docente que guíe la formación y el desarrollo profesional tanto al interior de las carreras como entre ellas (Caena, 2011).

\subsection{La sintonía en las competencias específicas en los estudios de casos}

La pregunta sobre la sintonía en las competencias específicas de los currículos renovados de pedagogía en educación general básica en instituciones universitarias con y sin proyecto Mecesup, se respondió desde los tres estudios de caso, aunque sólo los casos A y B definen competencias específicas, el caso C lo hace desde la definición de capacidades/habilidades que para estos efectos pueden ser equiparables, toda vez que construyen sentidos que persiguen la misma finalidad. Dado que los resultados distinguieron claramente dos ámbitos de formación: una, la de formación general de profesorado, y la otra, la de formación del trabajo pedagógico, dicha pregunta será respondida desde ambos ámbitos.

La sintonía en el ámbito de formación general de profesorado. Los resultados encontrados en el ámbito de formación general de profesorado se presentan en la tabla III y recogen tanto las unidades de análisis encontradas (objetivo 1) como la estructura identificada en cada competencia (objetivo 2) en este ámbito de formación. 
Tabla III. Competencias específicas en los casos A, B, C en formación general del profesorado

\begin{tabular}{|c|c|c|c|c|c|c|}
\hline & \multicolumn{2}{|c|}{ Caso A con Mecesup } & \multicolumn{2}{|c|}{ Caso B con Mecesup } & \multicolumn{2}{|c|}{ Caso C sin Mecesup } \\
\hline $\mathrm{N}^{\circ}$ & $\begin{array}{l}\text { Unidad de } \\
\text { Análisis }\end{array}$ & $\begin{array}{l}\text { Estructura de } \\
\text { competencia }\end{array}$ & $\begin{array}{l}\text { Unidad de } \\
\text { Análisis }\end{array}$ & $\begin{array}{l}\text { Estructura de } \\
\text { competencia }\end{array}$ & $\begin{array}{l}\text { Unidad de } \\
\text { Análisis }\end{array}$ & $\begin{array}{l}\text { Estructura de } \\
\text { competencia }\end{array}$ \\
\hline 1 & $\begin{array}{l}\text { Dominio de } \\
\text { fundamentos } \\
\text { teóricos } \\
\text { Ciencias de la } \\
\text { Educación }\end{array}$ & $\begin{array}{l}\text { SABER; } \\
\text { SABER HACER }\end{array}$ & $\begin{array}{l}\text { Genera } \\
\text { soluciones } \\
\text { desde los } \\
\text { fundamentos } \\
\text { teóricos de } \\
\text { Ciencias de la } \\
\text { Educación }\end{array}$ & $\begin{array}{l}\text { SABER } \\
\text { HACER; } \\
\text { SABER }\end{array}$ & $\begin{array}{l}\text { Utiliza los } \\
\text { fundamentos } \\
\text { teóricos de } \\
\text { Ciencias de la } \\
\text { Educación para } \\
\text { comprender la } \\
\text { realidad }\end{array}$ & $\begin{array}{l}\text { SABER } \\
\text { HACER; } \\
\text { SABER }\end{array}$ \\
\hline 2 & $s / i$ & $s / i$ & $\begin{array}{l}\text { Evaluación } \\
\text { crítica de } \\
\text { teorías y } \\
\text { currículum }\end{array}$ & $\begin{array}{l}\text { SABER } \\
\text { HACER; } \\
\text { SABER }\end{array}$ & $\begin{array}{l}\text { Reflexión crítica } \\
\text { del quehacer } \\
\text { desde teorías }\end{array}$ & $\begin{array}{l}\text { SABER } \\
\text { HACER; } \\
\text { SABER }\end{array}$ \\
\hline 3 & $\begin{array}{l}\text { Aporte al } \\
\text { conocimiento } \\
\text { desde la } \\
\text { investigación }\end{array}$ & $\begin{array}{l}\text { SABER ; } \\
\text { SABER HACER }\end{array}$ & $\begin{array}{l}\text { Generación } \\
\text { conocimiento a } \\
\text { partir de la } \\
\text { investigación }\end{array}$ & $\begin{array}{l}\text { SABER } \\
\text { HACER }\end{array}$ & $\begin{array}{l}\text { Uso de } \\
\text { estrategias } \\
\text { investigativas } \\
\text { para innovar }\end{array}$ & $\begin{array}{l}\text { SABER } \\
\text { HACER }\end{array}$ \\
\hline 4 & $\begin{array}{l}\text { Integración de } \\
\text { TIC }\end{array}$ & $\begin{array}{l}\text { SABER; } \\
\text { SABER HACER }\end{array}$ & $s / i$ & $\mathrm{~s} / \mathrm{i}$ & Uso de TIC & $\begin{array}{l}\text { SABER } \\
\text { HACER }\end{array}$ \\
\hline 5 & $s / i$ & $s / i$ & $s / i$ & $\mathrm{~s} / \mathrm{i}$ & $\begin{array}{l}\text { Integración de } \\
\text { recursos } \\
\text { tecnológicos }\end{array}$ & $\begin{array}{l}\text { SABER } \\
\text { HACER }\end{array}$ \\
\hline 6 & $\begin{array}{l}\text { Análisis del } \\
\text { sistema } \\
\text { educacional en } \\
\text { el contexto de } \\
\text { las políticas } \\
\text { educativas, la } \\
\text { gestión, ética y } \\
\text { responsabilidad } \\
\text { es profesionales }\end{array}$ & $\begin{array}{l}\text { SABER; SER; } \\
\text { SABER HACER }\end{array}$ & $s / i$ & $\mathrm{~s} / \mathrm{i}$ & $\begin{array}{l}\text { Relación del } \\
\text { sistema } \\
\text { educacional en } \\
\text { el contexto de } \\
\text { las políticas } \\
\text { educativas con } \\
\text { la labor } \\
\text { pedagógica }\end{array}$ & $\begin{array}{l}\text { SABER } \\
\text { HACER }\end{array}$ \\
\hline 7 & s/i & $s / i$ & $s / i$ & $s / i$ & $\begin{array}{l}\text { Reconoce } \\
\text { fortalezas y } \\
\text { debilidades } \\
\text { personal y } \\
\text { profesional }\end{array}$ & SER \\
\hline 8 & $\mathrm{~s} / \mathrm{i}$ & $\mathrm{s} / \mathrm{i}$ & $s / i$ & $\mathrm{~s} / \mathrm{i}$ & $\begin{array}{l}\text { Cumplimiento } \\
\text { de la gestión } \\
\text { escolar }\end{array}$ & $\begin{array}{l}\text { SABER } \\
\text { HACER }\end{array}$ \\
\hline 9 & $s / i$ & $s / i$ & $s / i$ & $\mathrm{~s} / \mathrm{i}$ & $\begin{array}{l}\text { Realiza su } \\
\text { trabajo con } \\
\text { responsabilidad } \\
\text { profesional }\end{array}$ & SER; SABER \\
\hline
\end{tabular}

A nivel general, los resultados evidencian una diferencia numérica de competencias específicas entre los casos A, B y C, a saber, 4, 3 y 9, respectivamente. Asimismo, es posible observar una mayor fragmentación en el caso $C$ que en A y B. En lo referente a relación con las unidades de análisis, los resultados muestran que la competencia 1 presente en todos los casos, si bien refiere al mismo contenido se construye desde habilidades muy diferentes (domina, genera soluciones, usa el saber para comprender la realidad). La competencia 2, presente en los casos B y C, se diferencian notoriamente toda vez que una refiere a la evaluación crítica de las teorías, la otra a reflexionar críticamente sobre el quehacer docente desde las teorías. La competencia 3, presente en todos los casos, refiere a contenidos similares, pero en A y B refiere a producir conocimiento por medio de la investigación, en cambio en $C$ al uso de estrategias investigativas. Por su parte, la competencia 4 de $A$, encuentra relación con 4 y 5 de $C$ y en ambos casos se corresponden. Finalmente, la competencia 6 del caso A, se relaciona con 6, 7, 8 y 9 del caso C, aunque difieren a nivel de significado, ya que en el caso $A$ se centra en análisis del sistema educacional en el marco de las políticas educativas y el desarrollo de la profesión, en cambio el caso C lo hace vinculando 
este marco referencial con la labor pedagógica propiamente.

Respecto a la relación con la estructura de cada competencia, éstas no cumplen los requisitos básicos de las tres dimensiones. Así, en el caso $B$ de las 4 competencias que define, solo 1 cumple las tres dimensiones, las otras sólo tienen dos; en el caso B, de las 3 competencias, ninguna cumple el criterio, 2 tienen dos dimensiones, y la última sólo una; en el caso $C$, de las 9 competencias, ninguna de ellas cumple la estructura, 3 de ellas tienen dos dimensiones y las otras 7 tienen sólo una. De las 16 competencias analizadas, 15 presentan la dimensión de "saber hacer" y en 8 es la única dimensión que presentan (7 de éstas corresponden al caso C). Así también, se observa que cuando se analizan las duplas de dimensiones, el "saber" se asocia al "saber hacer" en todos los casos, pero en A el "saber" hegemoniza el "saber hacer", en cambio en B y C, el "saber hacer" hegemoniza el "saber" quedando omitido el "ser" de la construcción de las competencias (a excepción de dos de ellas).

En cuanto a la relación con el objetivo 3 que persigue identificar las corrientes curriculares en los diseños innovados de las carreras de pedagogía general básica, desde los datos reportados en los objetivos 1 y 2 (tabla IV).

Tabla IV. Corrientes curriculares en los casos A, B y C en formación general del profesorado

\begin{tabular}{|c|c|c|c|}
\hline Indicadores & Caso A con Mecesup & Caso B con Mecesup & Caso C Sin Mecesup \\
\hline $\begin{array}{l}\text { Estructura de la } \\
\text { competencia } \\
\text { (dimensiones) }\end{array}$ & $\begin{array}{l}\text { No cumple } \\
\text { Presencia de dos y tres } \\
\text { dimensiones } \\
\text { (saber-saber hacer) }\end{array}$ & $\begin{array}{l}\text { No cumple } \\
\text { Presencia de dos y una } \\
\text { dimensión } \\
\text { (saber hacer-saber) }\end{array}$ & $\begin{array}{l}\text { No cumple } \\
\text { Presencia de una y dos } \\
\text { dimensiones } \\
\text { (saber hacer) }\end{array}$ \\
\hline Unidades de análisis & $\begin{array}{l}\text { Unidades de significados } \\
\text { centradas en contenidos } \\
\text { en contextos }\end{array}$ & $\begin{array}{l}\text { Unidades de significados } \\
\text { centradas en habilidades } \\
\text { asociadas a contenidos }\end{array}$ & $\begin{array}{l}\text { Unidades de significados } \\
\text { centradas en habilidades } \\
\text { asociadas a recursos y/o } \\
\text { productos }\end{array}$ \\
\hline $\begin{array}{l}\text { Características de las } \\
\text { competencias específicas }\end{array}$ & $\begin{array}{l}\text { Centradas en el } \\
\text { contenido temático } \\
\text { Situadas en algún } \\
\text { contexto }\end{array}$ & $\begin{array}{l}\text { Centradas en una } \\
\text { habilidad } \\
\text { Situadas en el campo } \\
\text { temático }\end{array}$ & $\begin{array}{l}\text { Centradas en el } \\
\text { resultado de aprendizaje } \\
\text { Situadas en recursos }\end{array}$ \\
\hline
\end{tabular}

Estos hallazgos permiten identificar que el caso A aún mantiene algunas coincidencias con la corriente curricular de orientación constructivista, como se observó en el momento de analizar las competencias generales, pues a nivel de las características de las competencias, éstas muestran contenidos situados en contextos, de la misma forma los significados que se construyen resultan coincidentes con ellas; sin embargo, se empieza a difuminar esta corriente cuando la estructura de cada competencia no recoge las tres dimensiones, y sobre todo deja de lado el "ser". En el caso B se fortalece su coincidencia con la visión funcionalista del currículum pues sus competencias siguen sin considerar el contexto y se centran mayoritariamente en la habilidad seguida del contenido (Joannert et al., 2008), lo cual podría encontrar relación con un enfoque racionalista académico (Soto, 2003), así tampoco dan cuenta de la estructura mínima exigida para definir una competencia desde las tres dimensiones (Rué y Martínez, 2005). Por su parte, el caso C es aún más determinante la impronta del funcionalismo, pues es posible identificar rasgos del currículum tecnológico, toda vez que tanto los significados que construyen las competencias como las características de las mismas vinculan habilidad con recurso (Soto, 2003). Es decir, se podría concluir que tanto los casos B como C, fueron diseñados desde el funcionalismo, pero desde enfoques diferentes.

Por consiguiente, la sintonización desde un análisis del modelo basado en competencia requiere de 8 competencias especializadas consideradas claves (Caena, 2011); de ellas, en A, B y C sólo cumplen con la de investigación, en cambio, A y C lo hacen además con la de uso de TIC, y C suma la de innovación. Es decir, los niveles de cumplimiento son excesivamente bajos en los tres casos, sin observar diferencias notorias entre aquellas carreras con o sin Mecesup.

Los niveles de sintonía en la formación para el trabajo pedagógico. Los resultados encontrados en el área de formación para el trabajo pedagógico, se presentan en la tabla $\vee$ y recogen tanto las unidades de 
análisis encontradas (objetivo 1) como la estructura identificada en cada competencia (objetivo 2) de este ámbito de formación.

Tabla V. Competencias específicas en casos A, B, C en formación para el trabajo pedagógico

\begin{tabular}{|c|c|c|c|c|c|c|}
\hline & \multicolumn{2}{|c|}{ Caso A con Mecesup } & \multicolumn{2}{|c|}{ Caso B con Mecesup } & \multicolumn{2}{|l|}{ Caso C sin Mecesup } \\
\hline $\mathrm{N}^{\circ}$ & Unidad de Análisis & $\begin{array}{l}\text { Estructura } \\
\text { de compe- } \\
\text { tencia }\end{array}$ & Unidad de Análisis & $\begin{array}{l}\text { Estructura } \\
\text { de compe- } \\
\text { tencia }\end{array}$ & Unidad de Análisis & $\begin{array}{l}\text { Estructura } \\
\text { de compe- } \\
\text { tencia }\end{array}$ \\
\hline 1 & $\begin{array}{l}\text { Diseño de procesos } \\
\text { de E-A desde } \\
\text { teorías y contextos } \\
\text { socioculturales }\end{array}$ & $\begin{array}{l}\text { SABER } \\
\text { HACER; } \\
\text { SABER }\end{array}$ & $\begin{array}{l}\text { Diseño de procesos } \\
\text { de E-A desde teorías } \\
\text { y contextos } \\
\text { socioculturales }\end{array}$ & $\begin{array}{l}\text { SABER } \\
\text { HACER; } \\
\text { SABER }\end{array}$ & $\begin{array}{l}\text { Planificación de la E-A } \\
\text { desde teorías curriculares, } \\
\text { los alumnos y los recursos }\end{array}$ & $\begin{array}{l}\text { SABER } \\
\text { HACER; } \\
\text { SABER }\end{array}$ \\
\hline 2 & 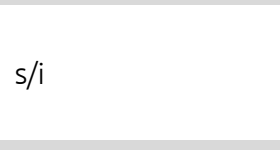 & $s / i$ & $\begin{array}{l}\text { Evaluación desde los } \\
\text { saberes disciplinares } \\
\text { y las particularidades } \\
\text { de los alumnos }\end{array}$ & $\begin{array}{l}\text { SABER } \\
\text { HACER; } \\
\text { SABER; } \\
\text { SER }\end{array}$ & $\begin{array}{l}\text { Evaluación desde los } \\
\text { saberes específicos y en } \\
\text { forma apropiada al } \\
\text { aprendizaje de los alumnos }\end{array}$ & $\begin{array}{l}\text { SABER } \\
\text { HACER; } \\
\text { SABER; } \\
\text { SER }\end{array}$ \\
\hline 3 & $s / i$ & $s / i$ & $\begin{array}{l}\text { Evaluación para la } \\
\text { mejora de los } \\
\text { aprendizajes }\end{array}$ & $\begin{array}{l}\text { SABER } \\
\text { HACER; } \\
\text { SER }\end{array}$ & $\begin{array}{l}\text { Evaluación para verificar el } \\
\text { logro de aprendizaje }\end{array}$ & $\begin{array}{l}\text { SABER } \\
\text { HACER }\end{array}$ \\
\hline 4 & $\mathrm{~s} / \mathrm{i}$ & $\mathrm{s} / \mathrm{i}$ & $\begin{array}{l}\text { Manejo de la } \\
\text { didáctica de la } \\
\text { especialidad y } \\
\text { currículum nacional } \\
\text { vigente }\end{array}$ & $\begin{array}{l}\text { SABER } \\
\text { HACER; } \\
\text { SABER }\end{array}$ & $\begin{array}{l}\text { Uso de recursos didácticos } \\
\text { para el logro de } \\
\text { aprendizajes desde } \\
\text { currículum nacional vigente }\end{array}$ & $\begin{array}{l}\text { SABER } \\
\text { HACER }\end{array}$ \\
\hline 5 & $s / i$ & $s / i$ & $s / i$ & $s / i$ & $\begin{array}{l}\text { Adecuaciones pedagógicas } \\
\text { a niveles y logros de } \\
\text { estudiantes }\end{array}$ & $\begin{array}{l}\text { SABER } \\
\text { HACER }\end{array}$ \\
\hline 6 & $\mathrm{~s} / \mathrm{i}$ & $\mathrm{s} / \mathrm{i}$ & $s / i$ & $s / i$ & $\begin{array}{l}\text { Categorización del } \\
\text { alumnado para } \\
\text { responsabilidad del éxito } \\
\text { escolar }\end{array}$ & $\begin{array}{l}\text { SABER } \\
\text { HACER; } \\
\text { SER }\end{array}$ \\
\hline 7 & $\begin{array}{l}\text { Diseño de } \\
\text { estrategias de } \\
\text { convivencia } \\
\text { democrática para el } \\
\text { desarrollo del } \\
\text { estudiante }\end{array}$ & $\begin{array}{l}\text { SABER } \\
\text { HACER; } \\
\text { SER }\end{array}$ & $s / i$ & $s / i$ & $\begin{array}{l}\text { Generación de clima de } \\
\text { aprendizaje para controlar } \\
\text { el conflicto }\end{array}$ & $\begin{array}{l}\text { SABER } \\
\text { HACER }\end{array}$ \\
\hline 8 & $s / i$ & $s / i$ & $\begin{array}{l}\text { Interacción con la } \\
\text { comunidad escolar } \\
\text { para optimizar el } \\
\text { desarrollo y los } \\
\text { aprendizajes de los } \\
\text { estudiantes }\end{array}$ & $\begin{array}{l}\text { SABER } \\
\text { HACER }\end{array}$ & $\begin{array}{l}\text { Identificación de la } \\
\text { trayectoria educativa del } \\
\text { estudiante para favorecer el } \\
\text { aprendizaje }\end{array}$ & $\begin{array}{l}\text { SABER } \\
\text { HACER }\end{array}$ \\
\hline 9 & $\mathrm{~s} / \mathrm{i}$ & $s / i$ & $s / i$ & $\mathrm{~s} / \mathrm{i}$ & $\begin{array}{l}\text { Sentido pedagógico de los } \\
\text { resultados }\end{array}$ & $\begin{array}{l}\text { SABER } \\
\text { HACER; } \\
\text { SER. }\end{array}$ \\
\hline 10 & $\begin{array}{l}\text { Dominio } \\
\text { disciplinario de la } \\
\text { enseñanza de } \\
\text { lenguaje en } \\
\text { acuerdo al } \\
\text { currículum nacional } \\
\text { y en diferentes } \\
\text { contextos sociales }\end{array}$ & $\begin{array}{l}\text { SABER } \\
\text { HACER; } \\
\text { SABER }\end{array}$ & $\begin{array}{l}\text { Reconstrucción del } \\
\text { saber disciplinario en } \\
\text { lenguaje para actuar } \\
\text { crítica y reflexiva de } \\
\text { su rol en la } \\
\text { construcción social y } \\
\text { cultural }\end{array}$ & $\begin{array}{l}\text { SABER } \\
\text { HACER- } \\
\text { SABER- } \\
\text { SER }\end{array}$ & $\begin{array}{l}\text { Dominio disciplinario en } \\
\text { lenguaje para enseñar en } \\
\text { acuerdo con el currículum } \\
\text { nacional }\end{array}$ & $\begin{array}{l}\text { SABER; } \\
\text { SABER } \\
\text { HACER }\end{array}$ \\
\hline 11 & $s / i$ & $s / i$ & $\begin{array}{l}\text { Competencias } \\
\text { comunicativas } \\
\text { adecuadas a } \\
\text { situaciones y } \\
\text { contextos }\end{array}$ & $\begin{array}{l}\text { SABER } \\
\text { HACER; } \\
\text { SER }\end{array}$ & $\begin{array}{l}\text { Comunicación oral y } \\
\text { escritura con efectividad y } \\
\text { corrección }\end{array}$ & $\begin{array}{l}\text { SABER } \\
\text { HACER; } \\
\text { SER }\end{array}$ \\
\hline 12 & $s / i$ & $s / i$ & $s / i$ & $s / i$ & $\begin{array}{l}\text { Valoración de la literatura y } \\
\text { arte para el desarrollo } \\
\text { personal y profesional }\end{array}$ & $\begin{array}{l}\text { SER; } \\
\text { SABER }\end{array}$ \\
\hline
\end{tabular}


A nivel general, los resultados evidencian una diferencia numérica de competencias específicas entre un caso y otro; así, el caso A define sólo 3, en cambio B define 7 , y C 12, situación que trae aparejada una diferencia de amplitud de contenidos; mientras el caso A es muy genérico cuando define el trabajo del profesorado, los casos B y C resultan notoriamente detallados.

En cuanto a la relación con las unidades de análisis, los resultados permiten evidenciar que la competencia 1 presente en todos los casos resulta muy próxima en cuanto a contenidos refiere. Una situación similar se observa en la competencia 2, presente sólo en los casos B y C, cuyos contenidos pueden considerarse equivalentes. En cambio, las competencias 3, 4, 8 y 11 de los casos B y C resultan notoriamente diferentes, mientras en la 3 de B se "evalúa para mejorar", en C se "evalúa para verificar", así la primera está centrada en el proceso, y la segunda en el producto; en la 4 de B se "domina la didáctica de la especialidad", en cambio en C se "usa recursos didácticos (generales)", la primera centrada en el contenido y la segunda centrada en el recurso; en la 8, mientras en B se centra en la interacción social como base para el desarrollo de los aprendizajes, en C lo hace en el diagnóstico; y en la 11, en B las competencias comunicativas se ajustan a situaciones y contextos, en cambio en $C$ son para demostrar efectividad y corrección en el dominio del lenguaje. Por su parte, la competencia 7, en A está centrada en el ejercicio del ambiente democrático, a diferencia de la condición de control del medio que tiene en C. Luego, la competencia 10 presente en los 3 casos estudiados, se observa que mientras A y C se centran en el dominio del lenguaje para dar cuenta del curriculum nacional, B reconstruye el lenguaje para actuar crítica y reflexivamente en la sociedad y la cultura. Finalmente, las competencias 5, 6, 9 y 12 sólo presentes en el caso C, no tienen punto de comparación con los otros casos.

Respecto a la relación con la estructura de cada competencia, los resultados evidencian que para el caso A ninguna cumple la exigencia de estructura, para el caso B sólo 2, y para el caso C sólo 1. Por el contrario, ponen el acento en dos dimensiones "saber hacer" y "saber" (en 13 de 22), seguidas de aquellas que cumplen una sola dimensión (en 6 de 22), finalmente, están también en forma minoritaria aquellas que incluyen el "ser" en su estructura (en 7 de 22).

Referente a la relación con el objetivo 3 que persigue identificar las corrientes curriculares en los diseños renovados de las carreras de Pedagogía general básica, desde los datos reportados en los objetivos 1 y 2, ver tabla VI.

Tabla VI. Corrientes curriculares en casos A, B y C formación para el trabajo pedagógico.

\begin{tabular}{l|l|l|l}
\hline Indicadores & Caso A con Mecesup & Caso B con Mecesup & Caso C Sin Mecesup \\
\hline $\begin{array}{l}\text { Estructura de la } \\
\text { competencia } \\
\text { (dimensiones) }\end{array}$ & $\begin{array}{l}\text { No cumple } \\
\text { Presencia de dos } \\
\text { dimensiones (saber- } \\
\text { saber hacer) } \\
\text { Unidades de } \\
\text { significados } \\
\text { centradas en } \\
\text { contenidos y } \\
\text { contextos }\end{array}$ & $\begin{array}{l}\text { Presencia de dos } \\
\text { dimensiones } \\
\text { (saber hacer-saber) }\end{array}$ & $\begin{array}{l}\text { No cumple } \\
\text { Presencia de una y dos } \\
\text { dimensiones } \\
\text { (saber hacer-ser) }\end{array}$ \\
Unidades de análisis & $\begin{array}{l}\text { centradas en habilidades } \\
\text { asociadas a contenidos y } \\
\text { contextos }\end{array}$ & $\begin{array}{l}\text { Unidades de significados } \\
\text { centradas en habilidades } \\
\text { asociadas a recursos, } \\
\text { productos y sujetos. }\end{array}$ \\
& $\begin{array}{l}\text { contenido temático } \\
\text { Situadas en algún } \\
\text { contexto }\end{array}$ & $\begin{array}{l}\text { Centradas en una habilidad } \\
\text { Situadas en el campo } \\
\text { temático } \\
\text { competencias espenticas de las }\end{array}$ & $\begin{array}{l}\text { Centradas en el resultado } \\
\text { Situadas en recursos } \\
\text { Situadas en el sujeto }\end{array}$ \\
\hline
\end{tabular}

En este ámbito de formación del trabajo pedagógico, los hallazgos siguen mostrando la tendencia encontrada en los análisis previos, el caso A mantiene algunos rasgos característicos de la corriente constructivista; sin embargo, se aleja de ésta cuando se le revisa desde la estructura de cada competencia (Joanert et al., 2008). Por su parte, en los casos B y C definen postura, pues mientras B se adscribe a una corriente praxiológica con un enfoque claramente socio-cognitivo centrado en el desarrollo de habilidades, contenido y contexto, el caso $\mathrm{C}$ se mantiene en una la corriente funcionalista, en un enfoque claramente tecnológico centrado en el desarrollo de contenido para conseguir resultados donde los 
recursos resultan fundamentales (Soto, 2003).

Por último, la sintonización en la formación para el trabajo pedagógico desde un análisis del modelo basado en competencia y sus 8 claves específicas (Caena, 2011) para desarrollarse en un mundo cambiante, está ausente en todos los casos, a excepción del caso B en que aparece una de ellas, la práctica reflexiva. Es decir, la sintonización entre aquellas carreras con o sin Mecesup no es posible de demostrar.

\section{Discusión y conclusiones}

La sintonización propuesta por la política Mecesup, que persigue una formación sintonizada con el mundo en cuanto a resultados de aprendizaje y demostración de competencias, es decir, una internacionalización de la formación en acuerdo con las corrientes principales de los actuales discursos de los países desarrollados, cabe ser discutida. Los resultados de esta investigación, aunque sólo pueden estar circunscritos a los estudios de casos presentados, muestran que no resulta posible demostrar tal sintonización entre las instituciones estudiadas. Una explicación posible a este hecho puede encontrarse en la figura de los asesores internacionales, quienes de alguna forma transmitieron sus propios modelos de formación más que una adscripción real al modelo basado en competencias, como propuso el modelo Tuning. Otra explicación puede circular en cuanto los diseñadores locales, quienes adscribieron al modelo basado en competencia a través del uso de un lenguaje que aparentemente recoge este tipo de diseño, pero que en la práctica plasmaron sus propias convicciones respecto del tipo de formación que persiguen. Y por último desde este nivel de discusión, los procesos de asesorías internacionales fracasaron en su esfuerzo por trasladar un modelo externo y escasamente conocido, profundizado, reflexionado y apropiado al interior de las instituciones universitarias formadoras, prevaleciendo con ello, la propia tradición institucional presente en cada grupo de diseñadores locales.

De esta forma, las realidades culturales, geográficas, lingüísticas propias de lo que se conoce como "primer mundo", se configuraron en un deber ser, alojado sólo a nivel discursivo, pero escasamente trasladó a los diseños de formación anclados en la realidad nacional, que frente a un proceso de homogenización y hegemonización del modelo basado en competencias impuesto desde el Estado como única realidad para acceder a los recursos económicos por parte de las instituciones formadoras, se confronta con una realidad portadora de estructuras y contenidos locales que configuran una diversidad de desempeños laborales muy distintos a aquellos de los países desarrollados, entre ellas, ruralidad en comunidad indígena, ruralidad en comunidades occidentales, urbanidad con y sin contexto indígena, ruralidad bilingüe y monolingüe, además, todas ellas en contextos de alta pobreza, cuestión que complejiza el desempeño (Ferrada y Turra, 2012; Catriquir, 2007; Gallego y Rodríguez, 2007; Harris y Chapman, 2004; Levine, 2006; Pinto, 2003). Todas ellas son temáticas ausentes en los actuales currículos de formación del profesorado. En la misma línea, investigaciones nacionales documentan y alertan sobre la necesidad de reconocer el saber acumulado en la experiencia del profesorado que se desempeña en diversidad de contextos, sobre todo de aquellos que lo hacen en escuelas en condiciones de pobreza, pues es allí donde se ve aumentada la complejidad del trabajo del docente, y es donde requiere contar con la mayor multiplicidad de capacidades para desenvolverse en la función que la sociedad le ha encomendado, a saber, lograr que sus estudiantes consigan aprender lo que la escuela pretende enseñar (Ferrada, 2001; Ferrada, Villena y Turra, 2015; Pinto, 2010; Pinto, 2008; Soto, 2008).

\section{Referencias}

Aneas, A. (2009) Competencias interculturales transversales: su diagnóstico en equipos de trabajo de baja cualificación. Revista de Investigación Educativa, 27(1), 105-123.

Astorga, B. (2017). Desde la Pedagógica Dialógica Enlazando Mundos, aportes para una Didáctica Dialógica en la Enseñanza de la Lengua (Tesis doctoral). Universidad Academia Humanismo Cristiano, Chile. 
Bravo, N. H. (2007). Competencias Proyecto Tuning-Europa, Tuning-América Latina. Informe de las cuatro reuniones del proyecto Tuning-Europa-América Latina. Recuperado de http://www.cca.org.mx/profesores/cursos/hmfbcp ut/pdfs/m1/competencias provectotuning.pdf

Caena, F. (2011). Literature review teachers' core competences: requirements and development. In: Education and Training 2020 Thematic Working Group 'Professional development of teachers. Recuperado de http://ec.europa.eu/dgs/education culture/repository/education/policy/strategicframework/doc/teacher-competences en.pdf

Catriquir, D. (2007). Mapunzugun: Una contribución al reposicionamiento de la denominación de la lengua y la sociedad mapunche. En T. Durán, D. Catriquir y A. Hernández (Eds.), Patrimonio cultural mapunche (V. I, pp. 35-51). Temuco: UCT.

De la Vega, L. (2015). Educational accountability: high and low points of its implementation and challenges for Latin America. Estudios sobre Educación, 29, 191-213. doi:10.15581/004.29.191-213

Del Valle, R., Kri, F. y Segovia, N. (2013). Estado de la movilidad estudiantil en las universidades del CRUCh. Recuperado de http://sct-chile.consejoderectores.cl/documentos WEB/Sistema de creditos transferibles/Santiago 2013/6.\%20Movilidad Estudiantil en SCT-Chile Pamela Ibarra.pdf

Estebaranz, A. (1994). Didáctica e innovación curricular. Universidad de Sevilla.

Ferrada y Del Pino, M. (2017): Dialogic-kishu kimkelay ta che educational research: participatory action research. Educational Action Research, 26(4). doi:10.1080/09650792.2017.1379422

Ferrada, D. (2001). Currículum crítico comunicativo. Barcelona: El Roure.

Ferrada, D. y Turra, O. (2012). Las comunidades de profesores como agentes legítimos en la construcción curricular para la formación inicial. Revista de Pedagogía Crítica Paulo Freire, 11(11), 207-217.

Ferrada, D., Villena, A. y Turra, O. (2015). Transformar la formación. Las voces del profesorado. Santiago de Chile: RIL Editores.

Ferrada, D., Villena, A., Catriquir, D., Pozo, G., Turra, O., Schilling, C. y Del Pino, M. (2014). Investigación dialógica-kishu kimkelay ta che en educación. Revista REXE, 13(26), 33-50.

Gallego, J. L. y Rodríguez, A. (2007). Tendencias en la formación inicial del profesorado en Educación Especial. Revista Electrónica Iberoamericana sobre la Calidad, Eficacia y Cambio en Educación , 5 (1), 102-117.

George-Nascimento, J. (2015). La didáctica dialógica: construcción intersubjetiva de la didáctica enlazando mundos con inteligencias múltiples, por una comunidad de investigación en un aula combinada de escuela básica rural (Tesis de Magíster en Ciencias de la Educación), Universidad Católica de la Santísima Concepción, Chile.

González, J. y Wagenaar, R. (2003). Tuning educational structures in Europe (Informe Final, Fase 1). Bilbao: Universidad de Deusto.

Guba, E. y Lincoln, Y. (2012) Controversias paradigmáticas, contradicciones y confluencias emergentes. En N. Denzin y I. Lincoln (Eds.), Paradigmas y Perspectivas en disputa (pp. 38-78). Barcelona: Gedisa.

Harris, A. y Chapman, C. (2004). Towards differentiated improvement for schools in challenging circumstances. British Journal of Educational Studies, 54, 4, 417-431.

Hord, S. (1987). Evaluating educational innovation. Nueva York: Croom Helm. 
Jonnaert, Ph., Barrette, J., Masciotra, D. y Yaya, M. (2008). La competencia como organizadora de los programas de formación: hacia un desempeño competente. Profesorado. Revista de Currículum y Formación de Profesorado, 12, 3, 1-31.

Levine, A. (2006). Educating school teachers. Washington: The Education School Project.

Marcelo, C. (2000). El cambio educativo como objeto de estudio. En A. Estebaranz (Ed.), Construyendo el cambio: perspectivas y propuestas de innovación educativa (pp. 37-57). Universidad de Sevilla.

Márquez, M. (2012). El proceso de construcción curricular en el modelo de competencias. Estudio de casos del rediseño e implementación del ciclo básico en una carrera de medicina. (Tesis inédita de doctorado). Pontifica Universidad Católica de Chile.

Mineduc. (2013). Programa de financiamiento por resultados de la educación terciaria- "Mecesup3". Manual Operacional. Recuperado de http://portales.mineduc.cl/usuarios/MECESUP/File/2013/ MECESUP\%203/ ManualOperacionalV5Julio2013.pdf

Mineduc. (2015). Programa Mecesup. Recuperado de

http://www.mecesup.cl/index2.php?id seccion=3586\&id portal=59\&id contenido=14892

Mingorance, P. y Estebaranz, A. (2009). Construyendo la comunidad que aprende: la vinculación efectiva entre la escuela y la comunidad. Revista Fuentes, 9, 179-199.

Noguera, P. (2003). City schools and the american dream. Reclaiming the promise of public education. Nueva York: Teachers College.

Pinto, R. (2003). El currículum necesario para una época compleja. Faro, 8. Recuperado de http://web.upla.cl/revistafaro/03 estudios/08 pinto.html

Pinto, R. (2008). El currículo crítico. Una pedagogía transformadora para la educación latinoamericana. Santiago: Ediciones PUC.

Pinto, R. (2010). En América Latina innovar en educación es posible gracias al esfuerzo crítico de sus educadores. Revista REXE, 9, 17, 65-85.

Rué, J. y Martínez, M. (2005). Les titulacions UAB en l'Espai Europeu d'Educació Superior [Las titulaciones UAB en el Espacio Europeo de Educación Superior]. Barcelona: IDES-UAB.

Soto, V. (2003) Paradigmas, naturaleza y funciones de la disciplina del curriculum. Revista Docencia, 20, 3646.

Soto, V. (2008). Los profesores en el territorio educativo y su evaluación desde el poder del estado subsidiario. Revista REXE, 7, 14, 25-35.

Stake, R. (1988). Investigación con estudios de casos. Madrid: Morata.

Townsend, T. (Ed.). (2007). International handbook of school effectiveness and improvement. Nueva York: Springer.

Valenzuela, J., Bellei, C. y De los Ríos, D. (2011). Segregación escolar en Chile. Santiago: Editorial puc/ UNESCO.

Zeichner, K. (2008). Settings for teacher education. In M. Cochran-Smith, S. Feiman-Nemser y D. Mc Intyre (Eds.), Handbook of Research on Teacher Education. Enduring questions in changing contexts. Nueva York/Abingdon: Routledge/Taylor \& Francis. 